\title{
DARK MATTER HALOS AS BOSE-EINSTEIN CONDENSATES
}

\author{
ECKEHARD W. MIELKE \\ Departamento de Física, \\ Universidad Autónoma Metropolitana-Iztapalapa, \\ Apartado Postal 55-534, C.P. 09340, México, D.F., MEXICO \\ E-mail address: ekke@xanum.uam.mx \\ BURKHARD FUCHS \\ Astronomisches Rechen-Institut, Mönchhofstr. 12-14, 69120 Heidelberg, Germany \\ E-mail address: fuchs@ari.uni-heidelberg.de \\ FRANZ E. SCHUNCK \\ Institut für Theoretische Physik, Universität zu Köln, 50923 Köln, Germany \\ E-mail address: fs@thp.uni-koeln.de
}

\begin{abstract}
Galactic dark matter is modelled by a scalar field in order to effectively modify Kepler's law without changing standard Newtonian gravity. In particular, a solvable toy model with a self-interaction $U(\Phi)$ borrowed from non-topological solitons produces already qualitatively correct rotation curves and scaling relations. Although relativistic effects in the halo are very small, we indicate corrections arising from the general relativistic formulation. Thereby, we can also probe the weak gravitational lensing of our soliton type halo. For cold scalar fields, it corresponds to a gravitationally confined Boson-Einstein condensate, but of galactic dimensions.
\end{abstract}

\section{Introduction}

The existence of dark matter was postulated for the first time by Zwicky 190 in 1933. He showed that the virial masses of clusters of galaxies exceed the mass of luminous matter by roughly one order of magnitude. In the 1970's spectrographs became sensitive enough that rotation curves of spiral galaxies could be observed in large numbers by Rubin and collaborators 6263 . They all showed a systematic tendency to stay flat as function of galactocentric radius, $v_{\varphi}(r) \simeq$ const., whereas the mass distributions of the bulges and the radially exponentially declining surface densities of the galactic disks lead to the prediction of falling rotation curves. Evidence for an extra mass component became even more decisive when HI-rotation curves 85 were observed, because the neutral hydrogen gas extends radially outwards many disk scale lengths. Even though there is a long debate 14 to what degree the inner parts of galaxies are dominated by dark matter, the flat outer HI-rotation curves prove 
beyond doubt the existence of dark matter halos. The analysis of the kinematics of satellite galaxies 88 showed that the dark halos extend out to several hundred kpc with the cumulative mass still rising linearly with galactocentric radius, $M(r) \propto r$, which corresponds to flat rotation curves.

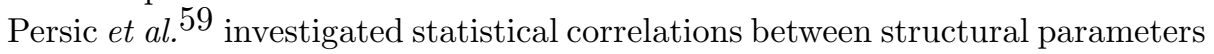
of visible and dark matter using a data set of almost 1000 galaxies and developed the model of the so called 'universal rotation curve'. Accordingly, galaxies of low luminosity are more dominated by dark matter than bright galaxies. Dwarf irregular galaxies and low surface brightness galaxies seem to be dominated completely by dark matter. Burkert ${ }^{17 / 18}$ finds for such galaxies an empirical fit to the rotation curves which implies asymptotically $v_{\varphi}^{2} \rightarrow \ln r / r$, i.e. a logarithmic modification of the Kepler law.

For several classes of gravitational theories, it has been argued that the introduction of dark matter is necessary 89 , although in the modified Newtonian dynamics (MOND) scheme, exact expressions were derived for the Newtonian potential and for the rotation curve 15 . However, recent observational constraints 1 make it more and more challenging to interpret galactic rotation in terms of new gravitational physics 10 .

An intriguing possibility is a scalar field model of the halo first proposed by one of us 6768697071 using a complex massless field coupled to the Einstein equation, an idea which was later taken up by several other authors. Hence, the complex massless scalar field forming star-like galactic halos embodies another form of dark energy.

Massive compact halo objects, so-called MACHOs2, consisting of baryonic matter or, alternatively, of boson stars (BSs) 47150178 do not seem to be sufficient to resolve this problem completely. More recent approaches 7330 propose connections between scalar field matter and the cosmological constant.

On the other hand, the standard cold dark matter (CDM) model, where dark matter particles interact only through gravity, may be in conflict with observational features on Mpc scale. In particular, the CDM model of Navarro, Frenk and White (NFW $\sqrt[55]{55}$ tends to predict density profiles of dark halos which are cuspy at the center $\left[53\right.$. Therefore, Spergel and Steinhardt $\frac{82}{2}$ proposed that dark matter is selfinteracting, an idea which found ramification in Refs. 61, 32. The evolution of the self-interacting dark matter has been recently analyzed in more detail 56 .

In the same vein, we consider here a mode 49151 where dark matter is modelled by a primordial scalar field with a self-interaction $U(\Phi)$. Since the observed rotation velocities are roughly bounded ${ }^{\mathrm{a}}$ by $v_{\varphi} \leq 10^{-3} c$, i.e. are non-relativistic, a Newtonian type approximation sufficies.

The formation of dark halos as a gigantic Bose-Einstein condensation in early ${ }^{a}$ The maximum velocity within a rotation curve of almost $400 \mathrm{~km} / \mathrm{s}$ is found in a Sa galaxy 62 ,
the velocity of $367 \mathrm{~km} / \mathrm{s}$ at the farest measured point belongs also to a Sa galaxy 
Universe is discussed in Sec. 2] In particular, a boson star like toy model with a $\Phi^{6}$ repulsive self-interaction is known $\underline{42}$ to allow in the limiting case of flat spacetime an exact spherically symmetric soliton solution of the corresponding nonlinear Klein-Gordon equation, as is shown in Sec. 3. Simulating the halo by such a nontopological soliton (NTS) for the positive range of the potential, yields a Newtonian mass distribution which provides a qualitatively rather good fit to the rotation curve data of dwarf irregular and low surface brightness galaxies, see Sec. 4 and

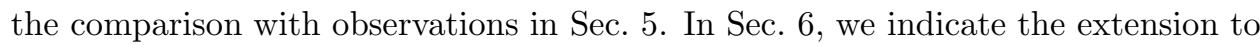
the general relativistic formulation and speculated on the superstring nature of the dark matter scalar. In Sec. 7 we display the gravitational lensing properties of the NTS halo.

\section{Dark halo as a self-gravitating Bose-Einstein condensate?}

Since Einstein and Bose it is well-known that identical integer spin particles can occupy the same ground state. Such a Bose-Einstein condensate (BEC) has been experimentally realized in 1995 for cold atoms of even number of electrons, protons, and neutrons, see Anglin and Ketterle ${ }^{33}$ for a review. In the mean-field ansatz, the interaction of the atoms in a dilute gas is approximated by the effective potential

$$
U(|\Psi|)_{\mathrm{eff}}=\frac{\lambda}{4}|\Psi|^{4} .
$$

This leads to a nonlinear Schrödinger equation for the macroscopic matter wave field $\Psi$, in this context known as Gross-Pitaevskii equation. In a microscopic approach, one introduces bosonic creation and annihilation operators $b^{\dagger}$ and $b$, respectively, satisfying $\left[b, b^{\dagger}\right]=1$, and finds that every number conserving normal ordered correlation function $\left\langle b_{1} \cdots b_{n}\right\rangle$ splits into the sum of all possible products of contractions $\left\langle b_{i} b_{j}\right\rangle$ as in Wick's theorem of quantum field theory (QFT). For $n=1$ one recovers the Gross-Pitaevskii equation, whereas the next order leads to Hartree-Fock-Bogoliubov equations 38 .

In the context of astrophysics, an assembly of massive real scalar particles has already been considered by Bonazzola and Pacin 13 in 1966. Later on, solutions for "systems of self-gravitating" bosons were considered by Ruffini and Bonazzola 64 similarly to the Hartree-Fock atom. Because a real scalar has no anti-particle states, the corresponding Klein-Gordon field $\Phi$ can only be decomposed into positive and negative frequency field operators:

$$
\Phi=\Phi^{+}+\Phi^{-}
$$

For a spherically symmetric configuration

$$
\begin{aligned}
\Phi^{+} & =\sum_{n l a} b_{n l a}^{\dagger} R_{l}^{n}(r) Y_{l}^{|a|}(\theta, \varphi) e^{-i \omega_{n l} t} \\
\Phi^{-} & =\sum_{n l a} b_{n l a} R_{l}^{n}(r)\left[Y_{l}^{|a|}(\theta, \varphi)\right]^{*} e^{+i \omega_{n l} t}
\end{aligned}
$$


can be expanded in terms of the wave function of a bound state similarly as for the hydrogen atom. Here $b_{n l a}$ and $b_{n^{\prime} l^{\prime} a^{\prime}}^{\dagger}$ are again creation and annihilation operators, $R_{l}^{n}(r)$ are radial distributions, $Y_{l}^{|a|}(\theta, \varphi)=(1 / \sqrt{4 \pi}) P_{l}^{|a|}(\cos \theta) e^{-i|a| \varphi}$ the spherical harmonics given in terms of the normalized Legendre polynomials, and $|a| \leq l$ are the quantum numbers of azimuthal and angular momentum. (The nodeless radial field solution $R_{0}^{0}(r)$ will later be denoted by $P(r)$.)

For the ground state of a cold configuration,

$$
|N\rangle=|N, 0,0, \ldots\rangle:=\prod_{1}^{N} b_{100}^{\dagger}|0\rangle
$$

is chosen 64 , where $|0\rangle$ is a vacuum state in the curved spacetime 'background'. The energy-momentum tensor $T_{\mu \nu}(\Phi)$ of the scalar field becomes now an operator. When coupled to gravity, the vacuum expectation value $\left\langle T_{\mu \nu}\right\rangle:=\left\langle N\left|: T_{\mu \nu}:\right| N\right\rangle$ for the ground state is inserted into the right-hand side of the Einstein equation (24), where : $T_{\mu \nu}$ : denotes normal ordering of the operator products.

An excited state $|N, n, l, a\rangle:=\Phi^{+}|0\rangle$ of positive energy can be obtained by applying creation operators. Such a 'gravitational atom ${ }^{[25]}$ represents a coherent quantum state, which nevertheless can have macroscopic size and large mass. The gravitational field is self-generated via the mean value $\left\langle T_{\mu \nu}\right\rangle$ of the energy-momentum tensor, but remains completely classical, whereas the real scalar is treated to some extent as operator.

Below some critical temperature (5), such configurations of coherent bosons would form Bose-Einstein condensates on an astrophysical scale ${ }^{7}$, and following T.D. Lee 41 are called boson stars (BSs).

Real scalars have the disadvantage that no local symmetry provides a conserved particle number, and so one needs to introduce by hand a normalization condition in order to stabilize the system.

It is gratifying to note that $B S s$ with repulsive self-interaction $U(|\Phi|)$ considered already by Mielke and Scherzer $\underline{44}$ and Colpi et al ${ }^{[20}$ have their counterparts in the effective potential (11) of BEC. Thus a cold BS can be considered as a selfgravitating BEC on an astrophysical scale 34378 . Moreover, BSs of rather different sizes can occur: it could be just a 'gravitational atom'; it could be as massive as the presumed black hole in the central part of a galaxy; or it could be an alternative explanation for parts of the dark matter in the halo of galaxies, as we are going to explain below. BSs, if they exist, would be an astrophysical realization with a self-generated gravitational confinement.

The idea of a cold mini-boson star as a BEC condensate on a galactic scale was reiterated, without referring to the earlier papers, in Refs. 34, 35] in a Newtonian approximation. A comparison of the Jeans scale of a dark matter halo and the de Broglie wave length of the gravitationally confined bosons provides the estimate $m_{\Phi} \simeq 10^{-22} \mathrm{eV} / \mathrm{c}^{2}$ for a tiny 'bare' mass of the bosons.

Bosonic particles such as fundamental scalars allow the possibility of a self- 
gravitating BEC on an astrophysical scale, commonly referred to as BS. However, a repulsive self-interaction has the physical effect of delaying the progressive collapse of a cosmic assembly formed by accretion. This, in particular, applies to a cold BS below the critical temperature

$$
T_{\mathrm{c}}=\frac{2 \pi \hbar^{2}}{m k}\left[\frac{N}{\xi(3 / 2) V}\right]
$$

where $\xi(z)$ is the Riemann zeta function, provided an ideal gas approximation is employed. However, for the possible formation of a BEC as a dark halo, one has to take into account its immersion into the heat bath of the cosmic background radiation of nowadays $2.726 \mathrm{~K}$.

The physical nature of gravitationally coupled scalar fields $\frac{16}{16}$ is at best speculative: From the field-theoretical point of view, a dilaton field $\varphi$ coupled universally to the trace of the energy-momentum tensor via

$$
L_{\mathrm{int}}:=\frac{1}{M_{\mathrm{Pl}}} \varphi T_{\mu}^{\mu}
$$

is a rather natural candidate. In order to apply this to galactic halos, one needs an ultralight scalar field with a Compton wave length $\lambda=\hbar / 2 m$ of the order of the galactic core $r_{\mathrm{c}} \simeq 10^{20} \mathrm{~m}$, corresponding to $m_{\varphi} \simeq 10^{-22} \mathrm{eV} / \mathrm{c}^{2}$. On the other hand, ultra-light 'gravi-scalars' arising in the framework of five-dimensional braneworlds are constrained 22 by the precisely observed slow-down of the Hulse-Taylor binary pulsar due the gravitational wave emission. The universal coupling (6) provides a natural mechanism 23 to protect such a tiny mass from renormalization due to quantum loops arising in the Standard Model. In order to suppress large long-range forces, an approximate global symmetry needs to be postulated which, nevertheless, allows a coupling of $\varphi$ to the Pontryagin term of electromagnetism via $\left(\varphi / M_{*}\right) F \wedge F$, cf. Carrol 19 .

A related proposal is that of Wetterich 87 which endows a massless dilaton (as a pseudo-Goldstone boson) with a tiny mass via the conformal anomaly. Such a dynamical scalar "quintessence" may also account for dark energy 4 . Another approach $\$ 56$ to unify clustered dark matter and dark energy is based on a BornInfeld type higher derivative Lagrangian for the same hypothetical scalar field.

Recently $\frac{73}{3}$, we analyzed the bifurcations in the mapping of a self-interacting scalar field $\Phi$ minimally coupled to Einstein's general relativity (GR) to a non-linear curvature scalar Lagrangian. Intriguingly, the higher-order Lagrangian bifurcates in almost linear Einsteinian patches, but with different effective gravitational strength and cosmological constant (dark energy) depending on the cosmological scale. More precise constraints on dark energy are expected to come from future data for the cosmic microwave background (CMB) radiation $\frac{4}{4}$, which may also get modified via the Sunyaev-Zeldovich effect during its long journey. 


\section{Newtonian non-topological solitons}

As a solvable toy model with self-interaction, let us consider the Klein-Gordon equation (25) with a $\Phi^{6}$ type potential

$$
U(|\Phi|)=m^{2}|\Phi|^{2}\left(1-\chi|\Phi|^{4}\right), \quad \chi|\Phi|^{4} \leq 1,
$$

where $m$ is the 'bare' mass of the boson and $\chi$ a coupling constant, which are thought of as constants of nature. The self-interaction in the radial Klein-Gordon equation takes the form $d U(P) / d P^{2}=m^{2}-3 m^{2} \chi P^{4}$. In flat spacetime, such a model was first considered by one of us $\frac{42}{4}$ for constructing non-topological soliton (NTS) solutions.

For a spherically symmetric configuration and the choice $\omega=m$, the corresponding nonlinear Klein-Gordon equation simplifies to an Emden type equation

$$
P^{\prime \prime}+\frac{2}{x} P^{\prime}+3 \chi P^{5}=0,
$$

familiar from the astrophysics of gaseous spheres. It has the completely regular exact solution

$$
P(r)= \pm \chi^{-1 / 4} \sqrt{\frac{A}{1+A^{2} x^{2}}},
$$

where we introduced the dimensionless radial coordinate $x:=m r$, and $A=$ $\sqrt{\chi} P^{2}(0)$ in terms of the central value. The solution depends essentially on the nonlinear coupling parameter $\chi$, since the limit $\chi \rightarrow 0$ would be singular. This feature is rather characteristic for soliton solutions. Already 1978, it has been generalized $\frac{42}{2}$ to a NTS with (quantized) angular momentum $l$.

In the following, it suffices to restrict ourselves to the above given range for which the potential $U(|\Phi|)$ remains positive. If effects of self-gravitation are taken into account, this scalar potential needs not to be bounded from below: In the case of general relativistic BSS4439407246/7648 with a self-interaction $\lambda|\Phi|^{4}$, it has been proven numerically $\frac{74}{4}$ that NTS exist even for negative values of $\lambda$. The extension to soliton stars $^{\mathrm{b}}$ have been considered by Lee and Pang 41 as well as Gleiser 31 .

The canonical energy-momentum tensor of a relativistic spherically symmetric scalar field is diagonal, i.e. $T_{\mu}{ }^{\nu}(\Phi)=\operatorname{diag}\left(\rho,-p_{r},-p_{\perp},-p_{\perp}\right)$ with

$$
\begin{aligned}
\rho & =\frac{1}{2}\left(\omega^{2} P^{2}+P^{\prime 2}+U\right), \\
p_{\mathrm{r}} & =\rho-U, \\
p_{\perp} & =p_{r}-P^{\prime 2},
\end{aligned}
$$

\footnotetext{
${ }^{\mathrm{b}}$ Our potential (7) is not bounded from below, however, its positive range is sufficient for applying our toy model. An example of a bounded potential is $U(|\Phi|)=m^{2}|\Phi|^{2}\left(1-|\Phi|^{2} /\left|\Phi_{0}\right|^{2}\right)^{2}$ typical for soliton stars, but then simple analytical expressions are not available and one needs, for large radius, to deal with asymptotic solutions like $P(r) \rightarrow \pm \sqrt{A /\left(1+B^{2} e^{2 x}\right)}$.
} 
where $^{\prime}=d / d r$. The form (10) is familiar from perfect fluids, except that the radial and tangential pressure generated by the scalar field are in general different, i.e. $p_{r} \neq p_{\perp}$. This anisotropy of scalar matter should not been ignored, since it holds even in flat spacetime, or in the Newtonian approximation.

From (10) we find in flat spacetime the energy-density

$$
\begin{aligned}
\rho & =\frac{m^{2}}{2}\left[2 P^{2}+P^{\prime 2}-\chi P^{6}\right] \\
& =\frac{A m^{2}}{2 \sqrt{\chi}\left(1+A^{2} x^{2}\right)}\left[2+\frac{A^{4} x^{2}-A^{2}}{\left(1+A^{2} x^{2}\right)^{2}}\right] .
\end{aligned}
$$

(If we would consider a real scalar field instead, there is merely the change of the first proportionality constant 2 in the bracket to 1.) The requirement of the positivity of $U(\Phi)$ at the origin yields the constraint $A<1$, which we will adopt in the following.

The leading term of the Newtonian type mass concentration (11) is exactly the density law of the quasi-isothermal sphere

$$
\rho(r) \simeq \frac{\rho_{0} r_{\mathrm{c}}^{2}}{r_{\mathrm{c}}^{2}+r^{2}} .
$$

At large radii the density falls of like $\rho \propto r^{-2}$ which corresponds to an asymptotically flat rotation curve. Comparing with the quasi-isothermal sphere (12), the central density of the NTS model is given by $\rho_{0} \simeq A m^{2} / \sqrt{\chi}$ and the core radius is $r_{\mathrm{c}} \simeq$ $1 / m A$. This implies a scaling law for the dark halos of the form

$$
\rho_{0} \simeq \frac{m}{\sqrt{\chi}} \frac{1}{r_{\mathrm{c}}} \propto \frac{1}{r_{\mathrm{c}}}
$$

where $A$, which may vary from halo to halo, cancels out.

This nonlinearly coupled scalar field excerts the following radial and tangential pressures:

$$
\begin{aligned}
p_{\mathrm{r}} & =\frac{m^{2}}{2}\left[\chi P^{6}+P^{\prime 2}\right]=\frac{A^{3} m^{2}}{2 \sqrt{\chi}\left(1+A^{2} x^{2}\right)^{2}} \simeq \frac{A^{3} m^{2}}{2 \sqrt{\chi}}, \\
p_{\perp} & =\frac{m^{2}}{2}\left[\chi P^{6}-P^{\prime 2}\right]=\frac{A^{3} m^{2}\left(1-A^{2} x^{2}\right)}{2 \sqrt{\chi}\left(1+A^{2} x^{2}\right)^{3}} \simeq \frac{A^{3} m^{2}}{2 \sqrt{\chi}}, \\
\rho+p_{\mathrm{r}} & =m^{2}\left[P^{2}+P^{\prime 2}\right] \\
& =\frac{A m^{2}}{\sqrt{\chi}\left(1+A^{2} x^{2}\right)}\left[1+\frac{A^{4} x^{2}}{\left(1+A^{2} x^{2}\right)^{2}}\right] .
\end{aligned}
$$

Thus, at the center of the NTS, we have $p_{\mathrm{r}}(0)=p_{\perp}(0)$. Asymptotically, we find at radial infinity

$$
p_{\mathrm{r}},-p_{\perp} \rightarrow \frac{m^{2}}{2 \sqrt{\chi} A x^{4}} .
$$

From the energy density (11) the mass function

$$
M(r):=\int_{0}^{r} \rho y^{2} d y
$$


can be obtained by straightforward integration. With the aid of REDUCE $\frac{66}{6}$ we find for our model:

$$
\begin{aligned}
M(r) & =\frac{1}{m A \sqrt{\chi}}\left[x+\frac{A^{2}-8}{8 A} \arctan (A x)-\frac{A^{2} x}{8} \frac{1+3 A^{2} x^{2}}{\left(1+A^{2} x^{2}\right)^{2}}\right], \\
& \simeq \frac{A}{6 \sqrt{\chi}} m^{2}\left(2-A^{2}\right) \cdot r^{3}, \\
& \rightarrow \frac{r}{A \sqrt{\chi}} .
\end{aligned}
$$

\section{Rotation curves}

The tangential velocity $v_{\varphi}$ of stars moving like "test particles" around the center of a galaxy is not directly measurable, but can be inferred from the redshift $z_{\infty}$ observed at spatial infinity, for which

$$
\left(1+z_{\infty}\right)^{2}=\frac{e^{\nu_{\infty}}}{e^{\nu}} \frac{\left(1 \pm v_{\varphi}\right)^{2}}{1-v_{\varphi}^{2}}
$$

holds. Due to their non-relativistic velocities in galaxies bounded approximately by $v_{\varphi} / c \leq 10^{-3}$, we observe $z_{\infty} \simeq v_{\varphi}$ (as first part of a geometric series) with the consequence that the lapse function necessarily tends to unity, i.e. $e^{\nu} \simeq e^{\nu_{\infty}} /\left(1-v_{\varphi}^{2}\right) \rightarrow 1$. In general, there are two dominating influences on the redshift of stars: The gravitational potential of all matter components within the galaxy (stars, gas, dark matter) and the Doppler effect. (Since for dwarf galaxies dark matter dominates up to the center, we can neglect the contribution of stars and gas.) If $e^{\nu_{\infty}}=1$, the redshift for receding stars is $z=e^{-\nu / 2} \sqrt{\left(1-v_{\varphi}\right) /\left(1+v_{\varphi}\right)}-1$. For example $\frac{70}{\text {, the influence }}$ of the gravitational field of the dark matter on the redshift is just about $1.5 \mathrm{~km} / \mathrm{s}$, hence, negligible. Of course, if the gravitational potential has the same order of magnitude as the Doppler effect, then a velocity of $300 \mathrm{~km} / \mathrm{s}$ which observations reveal so far, would be actually just $150 \mathrm{~km} / \mathrm{s}$, but this would certainly demand too strong gravitational potentials for galaxies.

In general, for the static spherically symmetric metric (27) considered lateron, an observer at rest at the equator of the Schwarzschild type coordinate system measures the following tangential velocity squared as a point particle (or a star, regarded as a sufficient small body 24 ) flies past him in its circular orbit, $c f$. Misner et al! $! 52$, p. 657 , Eq. (25.20):

$$
\begin{aligned}
v_{\varphi}^{2} & :=e^{-\nu} r^{2}\left(\frac{d \varphi}{d t}\right)^{2}=\frac{1}{2} r \nu^{\prime}=\frac{1}{2}\left[1-e^{-\lambda}+\kappa p_{\mathrm{r}} r^{2}\right] e^{\lambda} \\
& =\frac{\kappa}{2}\left[\frac{M(r)}{r}+p_{\mathrm{r}} r^{2}\right] \exp \left\{\frac{r}{r-\kappa M(r)}\right\} \\
& \simeq \frac{\kappa}{2}\left[\frac{M(r)}{r}+p_{\mathrm{r}} r^{2}\right] .
\end{aligned}
$$


Outside of matter $(\nu=-\lambda)$ for a weak gravitational field, Eq. (19) reduces to the Newtonian form $v_{\varphi, \text { Newt }}^{2} \simeq M(r) / r$. As is well-known 8684, a naive application of the Newtonian limit would have led us to geodesics in flat spacetime, i.e. as if gravity would not affect the motion of test bodies like our stars moving in the dark matter halo. Thus it is mandatory to go beyond, as is indicated by our approximation of the generally relativistic formula (19). Then, also the pressure component $p_{r} \neq 0$ of an anisotropic 'fluid' contributes, our prime example being the case of scalar fields. In our NTS model, however, due to the fast decrease of $p_{r}, c f$. Eq. (15), its contribution to the asymptotic value of the rotation velocity is almost negligible.

From the mass function (17) of the Newtonian NTS solution (9) and its radial pressure (14), we find for the rotation velocity

$$
v_{\varphi}^{2} / v_{\infty}^{2}=1+\left(\frac{A^{2}}{8}-1\right) \frac{\arctan (A x)}{A x}+\frac{A^{2}}{8} \frac{A^{2} x^{2}-1}{\left(1+A^{2} x^{2}\right)^{2}},
$$

for which the following approximations

$$
\begin{aligned}
& v_{\varphi}^{2} \simeq \frac{\kappa A\left(1+A^{2}\right) m^{2}}{6 \sqrt{\chi}} r^{2} \\
& v_{\varphi}^{2} \rightarrow v_{\infty}^{2}=\frac{\kappa}{2 A \sqrt{\chi}}=\frac{\kappa}{2 \chi P^{2}(0)} \leq 10^{-6},
\end{aligned}
$$

hold near the center and at the far field, respectively. Together with (17) we conclude that asymptotically $M \rightarrow 2 v_{\infty}^{2} r$.

Observationally, there is the rough restriction $v_{\varphi} / c \leq 10^{-3}$ of the rotation velocities of galaxies, which can be used to constrain the mass $m \leq 10^{-22} \mathrm{eV} / \mathrm{c}^{2}$ and the coupling constant $\chi$ of our NTS model.

\section{Astronomical tests}

The theoretical model predictions against rotation curve data of a set of low surface brightness galaxies taken from Ref. 11, 12 have recently been tested by Fuchs and Mielke [30] There the rotation curves of in total 54 galaxies have been measured with high resolution. For about half of them surface photometry is available. For these galaxies not only kinematical data have been provided, but also constructed dynamical models of the galaxies. The observed rotation curves are modelled as

$$
v_{\varphi}^{2}(r)=v_{\varphi, \text { bulge }}^{2}(r)+v_{\varphi, \text { disc }}^{2}(r)+v_{\varphi, \text { isgas }}^{2}(r)+v_{\varphi, \text { halo }}^{2}(r),
$$

where $v_{\varphi \text {, bulge }}, v_{\varphi, \text { disc }}, v_{\varphi \text {,isgas }}$, and $v_{\varphi \text {, halo }}$ denote the contributions due to the bulge, the stellar disc, the interstellar gas, and the dark halo, respectively. The radial variations of $v_{\mathrm{c}, \text { bulge }}(r), v_{\mathrm{c} \text {,disc }}(r)$, and $v_{\mathrm{c} \text {,isgas }}(r)$ were derived from the observations, while the normalizations by the mass-to-light ratios were left as free parameters of the fits of the mass models to the data.

Fits of the form (22) to observed rotation are notoriously ambiguous. Thus, for each galaxy several models are provided, one with zero bulge and disc mass, one model with a 'reasonable' mass-to-light ratio of the bulge and the disc, and finally 
a 'maximum-disc' model with bulge and disc masses at the maximum allowed by the data. Furthermore, for each galaxy two types of dark halo models are tried. One is the cusped NFW density law and the second is the quasi-isothermal sphere, whose observational consequences are recently been reviewed by Bosma 14 . While varying the disc contribution to the observed rotation curve leads to fits of the same quality, it is found that the quasi-isothermal sphere models of the dark halos give significantly better fits to the data than the cusped NFW density law. Thus the scalar field model presented here is in this aspect even superior to the cold dark matter model in its present form.

Prada et al ${ }^{60}$ used data from the Sloan digital sky survey on satellite galaxies of isolated host galaxies to probe on $100 \mathrm{kpc}$ scale the outer halo mass distributions. They find that the line-of-sight velocity dispersions of the satellites follow closely the radially declining velocity dispersion profile of halo particles in a NFW halo. This implies an outer mass density distribution of the form $\rho \propto r^{-3}$ which is at variance with the prediction of the quasi-isothermal sphere (12). In the cold dark matter model the system of satellite galaxies is assembled during the same accretion processes as the dark halo, and Prada et al .60 assume consistently for the satellites the same distribution function in phase space as for the halo particles. In the NTS model, however, the dark halo provides for the baryonic matter simply a Newtonian force field, for which self-gravity has been neglected in zeroth approximation. The distribution function of satellite galaxies in phase space is thus not specified and can be modelled according to the observations, even if the potential trough of the quasi-isothermal sphere has a shallower profile than a NFW halo.

Next we have examined the predicted scaling relation (131). A relation of this type was found empirically by Salucci and Burkert 65 , although this was based on the universal rotation curve model of Persic et al ${ }^{59}$ and the, also empirically derived, halo density profile of Burkert 17 . This density law resembles the quasiisothermal sphere in that it has also a homogeneous core. In Fig. 1 we show central densities versus the inverses of the core radii of quasi-isothermal dark halo models constructed in Refs. 11, 12 assuming for the discs mass-to-light ratios consistent with current population synthesis models. Despite some scatter, there is a clear correlation between $\rho_{0}$ and $r_{c}^{-1}$ over several orders of magnitude, $\log \left(\rho_{0}\right) \propto(1.46 \pm$ $0.55) \log \left(r_{c}^{-1}\right)$. Thus the dark halo model data seem to confirm statistically the scaling relation (13). The scatter in the correlation diagram is probably due to the near degeneracy of the fits to the observed rotation curves, even if the disc contributions to the rotation curves are fixed. Moreover, practice shows that the radial exponential scale lengths of the discs cannot be determined more precisely than about $20 \%$. This has a considerable effect for the disc contributions to the rotation curves (22) and, consequently, for the dark halo models and might also explain some of the scatter of the correlation diagram in Fig. 1] We believe, however, that this has not changed the general trend.

Using arguments of the density wave theory of galactic spiral structure, one of 


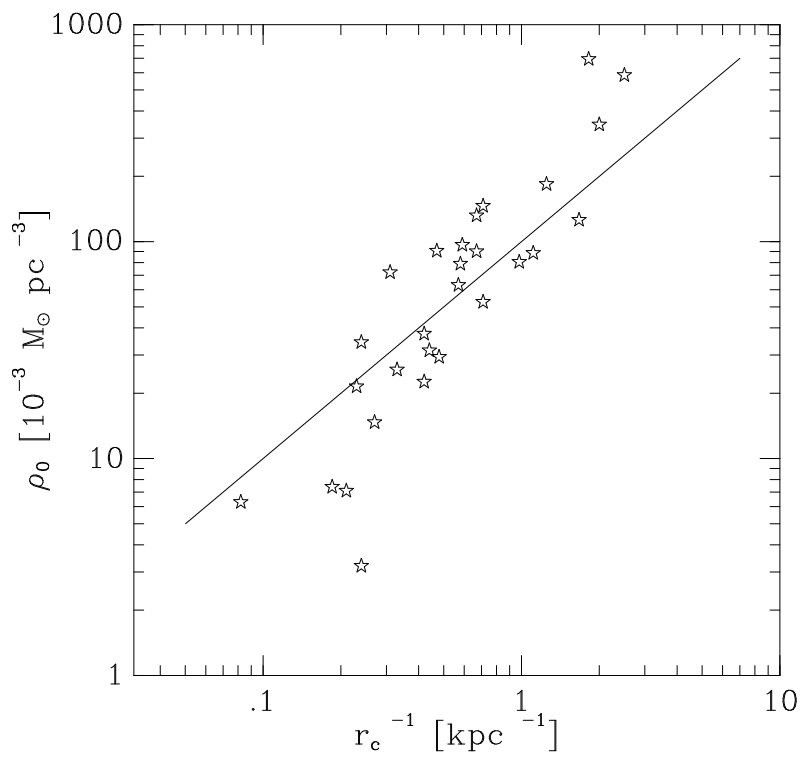

Figure 1. Central densities versus the inverses of the core radii of dark halos of low surface brightness galaxies derived from modelling their rotation curves. The halo models are constructed assuming 'realistic' mass-to-light ratios for the discs. The solid line is the predicted $\rho_{0} \propto r_{c}^{-1}$ relation.

us has pointed out, judging from the implied internal dynamics of the galactic discs, that the discs might be near to maximum 27/2829. This would imply for the discs of some galaxies mass-to-light ratios which are significantly higher than in current population synthesis models of the discs of low surface brightness galaxies. These can be modified, though, to yield higher mass-to-light ratios. Therefore we show in Fig. 2 the central densities versus the inverses of the core radii of the dark halo models, if they are constructed assuming 'maximum discs'. Although the dark halo parameters are shifted to other values, the linear correlation persists. With

$$
\log \left(\rho_{0}\right) \propto(1.08 \pm 0.39) \log \left(r_{c}^{-1}\right)
$$

the dark halo models fit nearly ideally to the predicted scaling relation (13). Included into Fig. 2 are also 'maximum disc' dark halo parameters of nearby bright galaxies 2629 which fit also well to the scaling law. We conclude from this discussion that the astronomical tests are rather encouraging for the scalar field model of dark halos presented here.

\section{General-relativistic corrections}

In the first part we were focused on a $\Phi^{6}$ toy model. In order to advance to more realistic models which include self-gravity, a full general-relativistic framework will 


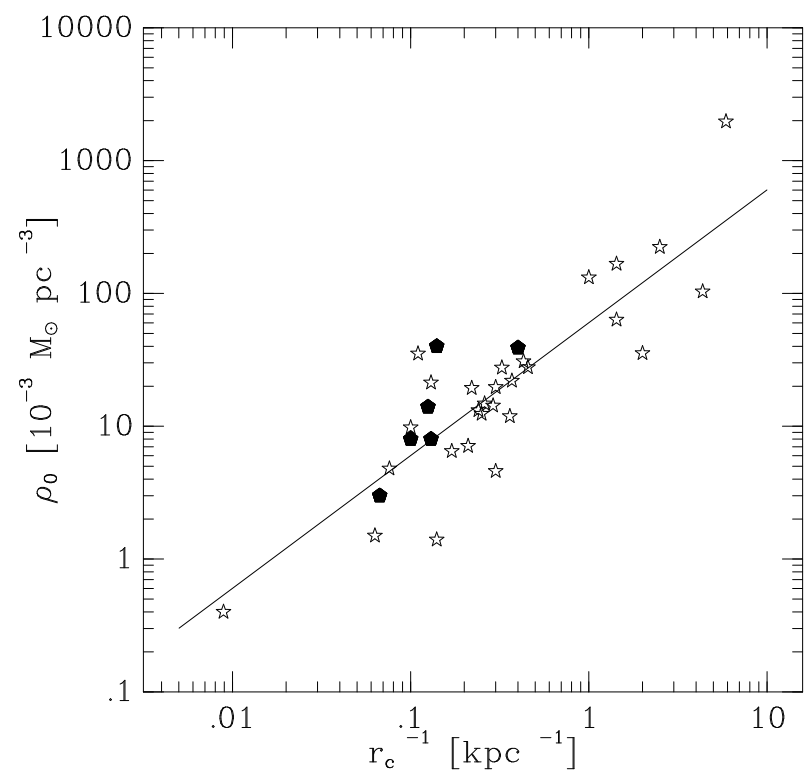

Figure 2. Same as Fig. 1 but the halo models are constructed assuming 'maximum discs'. Open symbols: low surface brightness galaxies, filled symbols: nearby bright galaxies.

be employed which departs from the coupled Einstein-Klein-Gordon equations

$$
\begin{aligned}
G_{\mu \nu}:=R_{\mu \nu}-\frac{1}{2} g_{\mu \nu} R & =-\kappa T_{\mu \nu}(\Phi), \\
\left(\square+\frac{d U}{d|\Phi|^{2}}\right) \Phi & =0 .
\end{aligned}
$$

Here $R$ is the curvature scalar, $\kappa=8 \pi G$, the gravitation constant ( $\hbar=c=1$ ), $g$ the determinant of the metric $g_{\mu \nu}$, and $\Phi$ a complex scalar field. Moreover, $\square:=(1 / \sqrt{|g|}) \partial_{\mu}\left(\sqrt{|g|} g^{\mu \nu} \partial_{\nu}\right)$ denotes the generally covariant d'Alembertian.

The stationarity ansatz

$$
\Phi(r, t)=P(r) e^{-i \omega t}
$$

describes a spherically symmetric bound state of the scalar field with frequency $\omega$. Note that the case of a real scalar field can readily be accommodated in our formalism by putting $\omega=0$ in our Ansatz.

For spherical symmetry of the halo, the line-element reads

$$
d s^{2}=e^{\nu(r)} d t^{2}-e^{\lambda(r)} d r^{2}-r^{2}\left(d \theta^{2}+\sin ^{2} \theta d \varphi^{2}\right),
$$

in which the functions $\nu=\nu(r)$ and $\lambda=\lambda(r)$ depend on the Schwarzschild type radial coordinate $r$. 
Then, the diagonal components of the energy-momentum tensor $T_{\mu}{ }^{\nu}(\Phi)=$ $\operatorname{diag}\left(\rho,-p_{r},-p_{\perp},-p_{\perp}\right)$ generalize to

$$
\begin{aligned}
\rho & =\frac{1}{2}\left(\omega^{2} P^{2} e^{-\nu}+P^{\prime 2} e^{-\lambda}+U\right), \\
p_{\mathrm{r}} & =\rho-U \\
p_{\perp} & =p_{r}-P^{\prime 2} e^{-\lambda},
\end{aligned}
$$

and the Emden equation (8) to the radial Klein-Gordon equation

$$
P^{\prime \prime}(r)+\left(\frac{\nu^{\prime}-\lambda^{\prime}}{2}+\frac{2}{r}\right) P^{\prime}(r)+e^{\lambda}\left(e^{-\nu} \omega^{2}-\frac{d U(P)}{d P^{2}}\right) P(r)=0 .
$$

The decisive non-vanishing components of the Einstein equation are the 'radial' equations

$$
\begin{aligned}
\nu^{\prime}+\lambda^{\prime} & =\kappa\left(\rho+p_{\mathrm{r}}\right) r e^{\lambda}, \\
\lambda^{\prime} & =\kappa \rho r e^{\lambda}-\frac{1}{r}\left(e^{\lambda}-1\right) .
\end{aligned}
$$

Two further components are identically fulfilled because of the contracted Bianchi identity $\nabla^{\mu} T_{\mu}{ }^{\nu} \equiv 0$ which is equivalent to the equation

$$
\frac{d}{d r} p_{\mathrm{r}}=-\nu^{\prime}\left(\rho+p_{\mathrm{r}}-\frac{2}{r}\left(p_{\mathrm{r}}-p_{\perp}\right)\right)
$$

of 'hydrostatic' equilibrium for an anisotropic fluid, a generalization $\underline{46}$ of the Tolman-Oppenheimer-Volkoff equation.

The general solution of Eq. (31) is

$$
e^{-\lambda}=1-\kappa \frac{M(r)}{r} \rightarrow 1-2 v_{\infty}^{2}
$$

where the mass function $M(r)$ is according to (16) only determined by the energy density $\rho$.

According to Eq. (17) of the NTS model, the radial metric component $e^{-\lambda}$ asymptotically approaches the value $1-2 v_{\infty}^{2}<1$. This is not unproblematic: After a redefinition of the radial coordinate $r \rightarrow \tilde{r}:=r / \sqrt{1-2 v_{\infty}^{2}}$, the asymptotic space has a deficit solid angle. The area of a sphere of radius $r$ is not $4 \pi r^{2}$, but $4 \pi\left(1-2 v_{\infty}^{2}\right) r^{2} ; c f$. analogous results for global monopoles and global textures $\frac{970}{}$. In order to avoid a linear increase of the mass function which would pose problems for the asymptotics, GR allows to redefine the radial coordinate. In the case of the more realistic phenomenological Burkert fit $\frac{17}{17}$ the velocity tends to zero at spatial infinity, with the consequence that no such deficit angle is to be expected.

So far we have considered a solvable model in flat spacetime. However, when the tangential velocity $v_{\varphi}=v_{\varphi}(x)$ is empirically known, we readily find from (19) 
that the metric components are in general given by

$$
\begin{aligned}
& e^{\nu}=\exp \left\{2 \int v_{\varphi}^{2} d \ln x\right\} \rightarrow x^{2 v_{\infty}^{2}}+K \\
& e^{\lambda}=\frac{1+2 v_{\varphi}^{2}}{1+\kappa p_{\mathrm{r}} r^{2}} \rightarrow 1+2 v_{\infty}^{2} \simeq\left(1-2 v_{\infty}^{2}\right)^{-1},
\end{aligned}
$$

where $K$ is a constant of integration; in the last line we used (33). The approximation for $e^{\lambda}$ is valid only for $p_{\mathrm{r}} r^{2} \simeq 0$, i.e. for non-singular radial pressure at the origin or for sufficiently fast decreasing pressure at infinity. Fortunately, in our NTS model, this condition is satisfied at the origin as well as at infinity, $c f$. the asymptotic function (15).

For our NTS rotation curve (20), we have derived the scalar field by setting the metric components to one in zeroth order Newtonian approximation. Then, we calculated the mass function and, consequently, the rotation curve. Now, we can do better and determine the metric potentials in first order approximation. For the lapse function we find

$$
\begin{gathered}
e^{\nu}=\exp \left\{\frac { v _ { \infty } ^ { 2 } } { 2 } \left[\ln \left\{x^{7} \sqrt{1+x^{2}}\left(\frac{1+A^{2} x^{2}}{A^{2} x^{2}}\right)^{7 / 2}\right\}\right.\right. \\
\left.\left.-\frac{1}{1+x^{2}}+\frac{7 A}{x} \arctan (A x)\right]\right\} .
\end{gathered}
$$

The shift function $e^{\lambda}$ is influenced by both, the rotation velocity (20) and the radial scalar field pressure (14). Therefore, a full account of self-gravity seems to demand numerical methods.

A more ambitious approach is to reconstruct a viable scalar potential $U(|\Phi|)$ from the empirical rotation curves on the basis of the Einstein equations, similarly as in the case of inflation 45 .

As a starting point, we may use the empirical Burkert profile ${ }^{17}$ which can be expressed in the following close analytical form:

$$
\begin{aligned}
v_{\varphi \mathrm{B}}^{2} / v_{\infty}^{2}=\frac{1}{2 x}\left\{\ln \left[(1+x)^{2}\left(1+x^{2}\right)\right]-2 \arctan (x)\right\} & \simeq 1-\frac{\arctan (x)}{x} \\
& \rightarrow 2 \frac{\ln x}{x}
\end{aligned}
$$

It has a maximum at $x=3.3$ in dimensionless units $x=r / r_{\mathrm{c}}$ and amounts at spatial infinity to a logarithmic modification of the Kepler law. Then, we find for the lapse function via REDUCE

$$
e^{\nu}=\exp \left\{\frac{v_{\infty}^{2}}{x}\left[x \ln \frac{1+x^{2}}{(1+x)^{2}}+2(1+x) \arctan (x)-\ln \left(1+2 x+2 x^{2}+2 x^{3}+x^{4}\right)\right]\right\}
$$

Assuming a negligible radial pressure term, i.e. $p_{\mathrm{r}} r^{2} \simeq 0$, the shift function $e^{\lambda}=$ $1+2 v_{\varphi}^{2}$ is simply determined by the Burkett rotation velocity (37). 
For this Burkert fit, we have attempted to reconstruc 49 a self-interaction potential $U(\Phi)=\rho-p_{\mathrm{r}}$ from the astronomical data, by resolving the radial Einstein equations (31) for $\rho$ and $p_{\mathrm{r}}$ with the general result

$$
U=\rho-p_{\mathrm{r}}=\frac{1}{\kappa r e^{\lambda}}\left[\lambda^{\prime}-\nu^{\prime}+\frac{2}{r}\left(e^{\lambda}-1\right)\right] .
$$

Accordingly, $U$ is positive when

$$
d \ln v_{\varphi} / d \ln x+v_{\varphi}^{2}+1 / 2 \geq 0
$$

holds, which is satisfied by the Burkert fit. However, since the radial dependence of the scalar field $\Phi(x)$ is still unknown, a numerical approach is needed in order to reconstruct the potential $U(\Phi)$ explicitly.

In view of the occurrence of scalar fields, like the dilaton or Kalb-Ramond axion in effective superstring models leading to axidilaton star 48 , the concordance of theory and observations in a viable self-interaction potential deserves further study.

\section{Gravitational lensing of dark halos}

Another promising approach to study the dark halos of galaxies is weak gravitational lensing, since it can be measured out to large projected distances from the lense. Hence, gravitational lensing of a transparent spherically symmetric NTS dark matter halo will be compared here with the scenario of a mini-BS lens 7721. We assume that the lens interior is almost empty of baryonic matter, such that deflected photons can travel freely through the space between the halo stars which embody only "small" disturbances 43 .

The deflection angle is then given by

$$
\hat{\alpha}\left(r_{0}\right)=2 \int_{r_{0}}^{\infty} \frac{b e^{\lambda / 2}}{r \sqrt{r^{2} e^{-\nu}-b^{2}}} d r-\pi,
$$

where $b=r_{0} \exp \left(-\nu\left(r_{0}\right)\right)$ is the impact parameter and $r_{0}$ denotes the closest distance between a light ray and the center of our halo. The lens equation for small deflection angles can be expressed as

$$
\sin (\theta-\beta)=\frac{D_{\mathrm{ls}}}{D_{\mathrm{os}}} \sin \hat{\alpha},
$$

where $D_{\mathrm{ls}}$ and $D_{\mathrm{os}}$ are the distances from the lens (deflector) to the source and from the observer to the source, respectively. The true angular position of the source is denoted by $\beta$, whereas $\theta$ stands for the image positions. One usually defines the reduced deflection angle to be $\alpha \equiv \theta-\beta=\sin ^{-1}\left(D_{\mathrm{ls}} \sin \hat{\alpha} / D_{\mathrm{os}}\right)$. However, equation (42) relies on substitution of the distance from the source to the point of minimal approach by the distance from the lens to the source $D_{\mathrm{ls}}$. For large deflection angles 


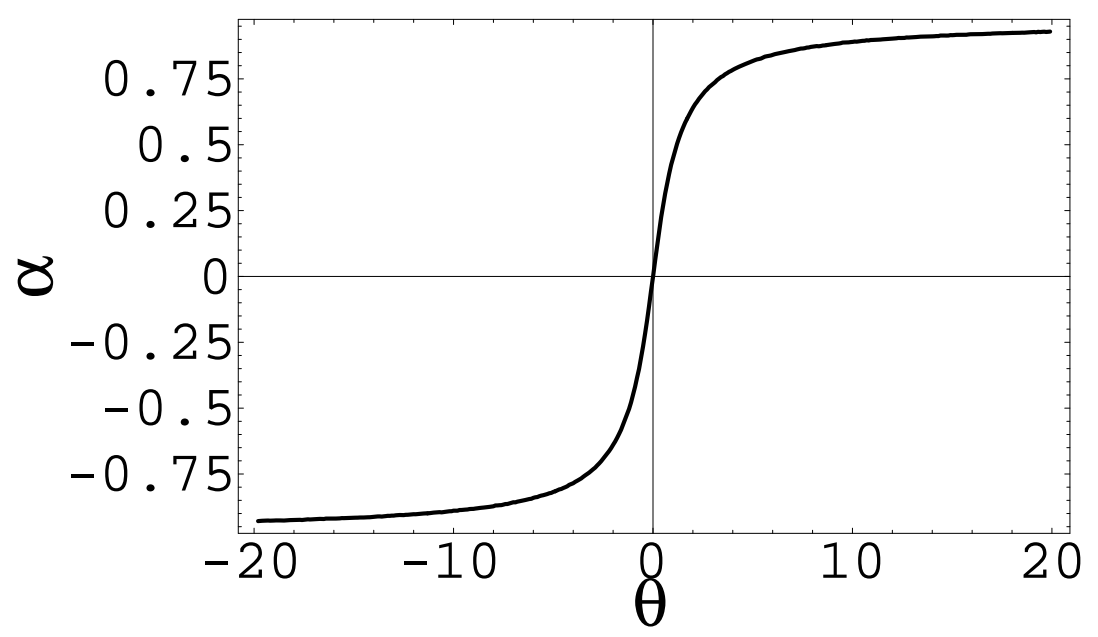

Figure 3. Reduced deflection angle for the NTS dark matter halo.

the distance $D_{\mathrm{ls}}$ cannot be considered a constant but it is a function of the deflection angle so that the form of the lens equation changes 21 into

$$
\sin \alpha=\frac{D_{\mathrm{ls}}}{D_{\mathrm{os}}} \cos \theta \cos \left[\operatorname{arsin}\left(\frac{D_{\mathrm{os}}}{D_{\mathrm{ls}}} \sin (\theta-\alpha)\right)\right][\tan \theta+\tan (\hat{\alpha}-\theta)] .
$$

The reduced deflection angle can be kept defined as $\alpha \equiv \theta-\beta$; cf. Ref. [58] with more details on the lens equation.

Since our dark matter halo, the NTS soliton, has only a weak gravitational field, we can apply (42) together with (41). The deflection angle is determined by the gravitational potentials (34), (35) which are functions of the NTS rotation curve (20) and the radial pressure (14). As an imput, we use the best fit value ${ }^{51}$ of $A=0.805$ to the Burkert profile (37). The asymptotic rotation velocity is taken to be $v_{\infty}=c / 1000$.

Numerical computations of the reduced deflection angle for the NTS halo were performed by assuming that the halo lens is half-way between the observer and the source, i.e. $D_{\mathrm{ls}} / D_{\mathrm{os}}=1 / 2$. The result is shown in Fig. [3] where $\alpha$ and $\theta$ are measured in arcsecond, but in general the units of $\theta$ depends on the oberserver-lens distance (further details, see Refs. 21, 79). We recognize prominent features of the NTS lens due to the linear increasing mass (17). There exists a limiting value of the reduced deflection angle of about $\alpha=0.93$ arcsecond. Hence, if the true angular position of the source $\beta$ increases, nevertheless the halo lens deflects the light with a constant value. This behavior would change if the mass of the halo becomes finite.

We can compare this result with the strong gravitational source of a BS for different potentials $U$, when the BS lens is again half-way between the observer and the source 2179 . Observable differences are found depending on the choice 
of the self-interaction. In the case of a simple mass term $U(|\Phi|)=m^{2}|\Phi|^{2}$, the largest possible value of $\alpha$ is 23.03 degrees with an image at about $\theta=n \times 2.88$ arcsec where $n=n\left(D_{\mathrm{ol}}, \omega\right)$ is the distance factor which is a function of the distance from the observer to the lens and the scalar field frequency the inverse of which is associated with the BS radius. For non-relativistic BS approximations, smaller angles will occur. The angle $\theta$ of the image position can have very different orders of magnitude, depending on $n$. For example, $n=1$ fixes $\theta$ to be measured in arcsec. Under the assumption that the BS mass is $10^{10} M_{\odot}$, the distance $D_{\mathrm{ol}}$ is about 100 pc. If the distance factor is $n=10^{-3}$, then $\theta$ is measured in milli-arcsec and the BS-lens is at about $100 \mathrm{kpc}$ from the observer.

In comparison, halos modelled by the Burkert profile will not produce strong lensing as well 157 . Differences of our NTS model and the Burkert fit can be traced back to the fact that the metric corresponding to an asymptotically constant velocity $v_{\infty}$ in general exhibits a deficit angle similarly as a cosmic string 83 . The linear mass density peaks at the location of the string, thereby producing a strong lensing.

The properties of dark matter halos inferred from weak lensing 33 provides a strong support for the existence of dark matter, wheras alternative theories of gravity such as MOND can almost be excluded.

\section{Acknowledgments}

We would like to thank Fjodor Kusmartsev, Humberto Peralta and Remo Ruffini for helpful discussions and comments. One of us (F.E.S.) acknowledges research support provided by a personal fellowship. Moreover, (E.W.M.) acknowledges the support of SNI and thanks Noelia, Markus Gérard Erik, and Miryam Sophie Naomi for encouragement.

\section{References}

1. A. Aguirre, C. P. Burgess, A. Friedland and D. Nolte, Class. Quantum Grav. 18, R223 (2001).

2. C. Alcock et al. [MACHO Collaboration], Phys. Rev. Lett. 74, 2867 (1995).

3. J.R. Anglin and W. Ketterle, Nature 416, 211 (2002).

4. C. Baccigalupi, A. Balbi, S. Matarrese, F. Perrotta and N. Vittorio, Nucl. Phys. Proc. Suppl. 124, 68 (2003).

5. S. Balberg and S. L. Shapiro, Phys. Rev. Lett. 88, 101301 (2002).

6. S. Balberg, S. L. Shapiro and S. Inagaki, Astrophys. J. 568, 475 (2002).

7. S. Banik and D. Bandyopadhyay, J. Phys. G 28, 1949 (2002).

8. C. Barceló, S. Liberati and M. Visser, Class. Quantum Grav. 18, 1137 (2001).

9. M. Barriola and A. Vilenkin, Phys. Rev. Lett. 63, 341 (1989); N. Turok and D. Spergel, Phys. Rev. Lett. 64, 2736 (1990).

10. J. D. Bekenstein, Phys. Lett. B202, 497 (1988).

11. W.J.G. de Blok, S.S. McGaugh and V.C. Rubin, Astron. J. 122, 2396 (2001).

12. W.J.G. de Blok and A. Bosma, Astron. Astroph 385, 816 (2002).

13. S. Bonazzola and F. Pacini, Phys. Rev. 148, 1269 (1966). 
14. A. Bosma: "Dark matter in galaxies: Observational overview", Preprint astro-ph/0312154

15. R. Brada and M. Milgrom, Mon. Not. R. Astron. Soc. 276, 453 (1995).

16. C. H. Brans: "Gravity and the tenacious scalar field," in: On Einstein's Path. Essays in Honor of Engelbert Schucking, A. Harvey, ed. (Springer, New York, 1999) pp. 121 $-138$.

17. A. Burkert, Astrophys. J. 447, L25 (1995).

18. A. Burkert and J. Silk: "On the structure and nature of dark matter halos", Preprint astro-ph/9904159

19. S. M. Carroll, Phys. Rev. Lett. 81, 3067 (1998).

20. M. Colpi, S.L. Shapiro and I. Wasserman, Phys. Rev. Lett. 57, 2485 (1986).

21. M.P. Dąbrowski and F.E. Schunck, Astrophys. J. 535, 316 (2000).

22. R. Durrer and P. Kocian: "Testing braneworlds with the binary pulsar", Preprint hep-th/0305181

23. G. R. Dvali, G. Gabadadze and M. A. Shifman, Mod. Phys. Lett. A16, 513 (2001).

24. J. Ehlers and R. Geroch, Ann. Phys. (NY) 309, 232 (2004).

25. R. Ferrell and M. Gleiser, Phys. Rev. D40 2524 (1989).

26. B. Fuchs, ASP Conf. Ser. 182, 365 (1999).

27. B. Fuchs, in: Dark Matter in Astro- and Particle Physics, H. V. KlapdorKleingrothaus, and R. D. Viollier, eds. (Springer, Berlin 2002), 28.

28. B. Fuchs, in: Proceedings of the Fourth International Workshop on the Identification of Dark Matter, Neil J. C. Spooner and Vitaly Kudryavtsev, eds. (World Scientific, Singapore 2003), 72.

29. B. Fuchs, Astroph. Space Sci. 284, 719 (2003).

30. B. Fuchs and E.W. Mielke: "Scaling behaviour of a scalar field model of dark matter halos", Mon. Not. R. Astron. Soc. (2004), Preprint astro-ph/0401575

31. M. Gleiser, Phys. Rev. Lett. 63, 1199 (1989).

32. J. Goodman, New Astron. 5, 103 (2000).

33. H. Hoekstra, H. K. C. Yee and M. D. Gladders: "Properties of galaxy dark matter halos from weak lensing," Preprint astro-ph/0306515

34. W. Hu, R. Barkana and A. Gruzinov, Phys. Rev. Lett. 85, 1158 (2000).

35. W. Hu, R. Barkana and A. Gruzinov, "Cold and fuzzy dark matter", Preprint astro-ph/0003365

36. I.T. Iliev and P.R. Shapiro, Astrophys. J. 546, L5 (2001).

37. K.R.W. Jones and D. Bernstein, Class. Quantum Grav. 18, 1513 (2001).

38. Th. Köhler and K. Burnett, "A microscopic quantum dynamics approach to the dilute condensed Bose gas", Preprint cond-mat/0110594

39. F.V. Kusmartsev, E.W. Mielke and F.E. Schunck, Phys. Rev. D43, 3895 (1991).

40. F.V. Kusmartsev, E.W. Mielke and F.E. Schunck, Phys. Lett. A157, 465 (1991).

41. T.D. Lee and Y. Pang, Phys. Rep. 221, 251 (1992).

42. E.W. Mielke, Phys. Rev. D18, 4525 (1978); Lett. Nuovo Cim. 25, 424 (1979).

43. E.W. Mielke (2002): "Gamma rays from boson anti-boson star mergers", Proceedings of the ICGA-5, Gravitation \& Cosmology Supplements, Vol.8, Supplement II N2 (ICGA-5 Proceedings), pp. 111-113 (2002).

44. E.W. Mielke and R. Scherzer, Phys. Rev. D24, 2111 (1981).

45. E.W. Mielke and F.E. Schunck, Phys. Rev. D52, 672 (1995).

46. E.W. Mielke and F.E. Schunck (1996): "Rotating boson stars" in: Gravity, Particles and Space-Time, ed. by P. Pronin and G. Sardanashvily (World Scientific, Singapore 1996), pp. 391-420.

47. E.W. Mielke and F.E. Schunck, Nucl. Phys. B564, 185 (2000). 
48. E.W. Mielke and F.E. Schunck, Gen. Rel. Grav. 33, 805 (2001).

49. E.W. Mielke and F.E. Schunck, Phys. Rev. D66, 023503 (2002).

50. E.W. Mielke and F.E. Schunck (2002): "Boson and axion stars", (Chairperson's report) Proc. of the Ninth Marcel Grossman Meeting on General Relativity, Rome, 2000, V.G. Gurzadyan, R. Jantzen and R. Ruffini, eds. (World Scientific, Singapore, 2002), pp. 581-590.

51. E.W. Mielke, F.E. Schunck and H.H. Peralta, Gen. Rel. Grav. 34, 1919 (2002).

52. C.W. Misner, K.S. Thorne and J.A. Wheeler: Gravitation (W.H. Freeman, New York 1973).

53. B. Moore, T. Quinn, F. Governato, J.Stadel and G. Lake: "Cold collapse and the core catastrophe," Mon. Not. R. Astron. Soc. 310, 1147 (1999).

54. A. Myers, T. Shanks, Ph. Outram, B. Frith and A. Wolfendale: "Evidence for an extended Sunyaev-Zeldovich effect in WMAP data" Mon. Not. R. Astron. Soc. 347, L67 (2004).

55. J.F. Navarro, C.S. Frenk and S.D.M. White, Astroph. J. 490, 493 (1997).

56. T. Padmanabhan and T. R. Choudhury, Phys. Rev. D66, 081301 (2002).

57. Y. Park and H. C. Ferguson, Astrophys. J. 589, L65 (2003).

58. V. Perlick: "On the exact gravitational lens equation in spherically symmetric and static spacetimes", Preprint gr-qc/0307072

59. M. Persic, P. Salucci and F. Stel, Mont. Not. R. Astr. Soc. 281, 27 (1996).

60. F. Prada, M. Vitvitska, A. Klypin A. et al., Astroph. J. 598, 260 (2003).

61. A. Riotto and I. Tkachev, Phys. Lett. B484, 177 (2000).

62. V.C. Rubin, W.K. Ford, Jr. and N. Thonnard, Astrophys. J. 238, 471 (1980).

63. V.C. Rubin, D. Burstein, W.K. Ford, Jr. and N. Thonnard, Astrophys. J. 289, 81 (1985).

64. R. Ruffini and S. Bonazzola, Phys. Rev. 187, 1767 (1969).

65. P. Salucci and A. Burkert, Astrophys. J. 537, L9 (2000).

66. E. Schrüfer: "EXCALC: A system for doing calculations in the calculus of modern differential geometry", Preprint (GMD 1999).

67. F.E. Schunck, "A matter model for dark halos of galaxies and quasars", Preprint FNAL FPRINT-95-10 (1994).

68. F.E. Schunck: Selbstgravitierende bosonische Materie, Ph. D. thesis, University of Cologne (Cuvillier Verlag, Göttingen 1996).

69. F.E. Schunck, "Massless scalar field models rotation curves of galaxies" in: Aspects of Dark Matter in Astro- and Particle Physics, H.V. Klapdor-Kleingrothaus and Y. Ramachers, eds. (World Scientific, Singapore 1997), pp. 403-408.

70. F.E. Schunck, "A scalar field matter model for dark halos of galaxies and gravitational redshift", Preprint astro-ph/9802258

71. F.E. Schunck: "Boson halo: Scalar field model for dark halos of galaxies", Proc. of the Eigth Marcel Grossman Meeting on General Relativity, Jerusalem, 1997, T. Piran and R. Ruffini, eds. (World Scientific, Singapore 1999) pp. 1447-1449.

72. F.E. Schunck, F.V. Kusmartsev and E.W. Mielke, "Stability of charged boson stars and catastrophe theory" in: Approaches to Numerical Relativity, R. d'Inverno (ed.) (Cambridge University Press, Cambridge 1992), pp. 130-140.

73. F.E. Schunck, F.V. Kusmartsev and E.W. Mielke: "Dark matter problem and effective curvature Lagrangians", Preprint 2003.

74. F.E. Schunck and A.R. Liddle, Phys. Lett. B404, 25 (1997).

75. F.E. Schunck and E.W. Mielke: "Rotating boson stars" in Proceedings of the Bad Honnef Workshop Relativity and Scientific Computing: Computer Algebra, Numerics, Visualization, ed. F.W. Hehl, R.A. Puntigam and H. Ruder (Springer, Berlin 1996) 
pp. 8-11, 138-151.

76. F.E. Schunck and E.W. Mielke, Phys. Lett. A249, 389 (1998).

77. F.E. Schunck and E.W. Mielke, Gen. Rel. Grav. 31, 787 (1999).

78. F.E. Schunck and E.W. Mielke, Class. Quantum Grav. 20, R301 (2003).

79. F.E. Schunck and D. Torres, Int. J. Mod. Phys. D 9, 601 (2000).

80. D.W. Sciama: Modern cosmology and the dark matter problem (Cambridge, Cambridge University Press 1993).

81. P.R. Shapiro, I.T. Iliev and A.C. Raga, Mont. Not. R. Astr. Soc. 307, 203 (1999).

82. D.N. Spergel and P.J. Steinhardt, Phys. Rev. Lett. 84, 3760 (2000).

83. N. Straumann: "Cosmic strings and galaxy formation", Invited lecture given at the 2nd Workshop on High Energy Astrophysics, July 1987. Published in Ringberg Astrophys. 0174 (1987).

84. R. A. Sussman and X. Hernandez, Mon. Not. Roy. Astron. Soc. 345, 871 (2003).

85. T.S. van Albada, J.N. Bahcall. K. Begeman, R. Sancisi, Astroph. J. 295, 305 (1985).

86. R.M. Wald: General Relativity (University of Chicago Press, Chicago 1984), p. 78.

87. C. Wetterich, Phys. Rev. Lett. 90, 231302 (2003).

88. D. Zaritzky, R. Smith, C.S. Frenk and S.D.M. White, Preprint astro-ph/9611199

89. V.V. Zhytnikov and J.M. Nester, Phys. Rev. Lett. 73, 2950 (1994).

90. F. Zwicky, Helv. Phys. Acta 6, 110 (1933). 